\title{
Evaluación del impacto de una intervención educativa en el campo de la tanatología en el INNN
}

\author{
María del R. Durán Medina, Jesús Ramírez Bermúdez, Norma Cruz, Maldonado, Calixto Emmanuelle Patiño Mora, \\ Aída Valero Chávez.
}

RESUMEN

Objetivo: evaluar si una intervención educativa en el área de la tanatología modifica la percepción de los prestadores de servicios de salud en el Instituto. Existe un cierto desconocimiento general sobre el tema; por lo tanto, el saber que es la tanatología ayudará al equipo de salud a brindar atención integral al paciente, cubriendo todos los aspectos que conforman a un individuo, fisiológico, psicológico, emocional, social y espiritual; con calidez y calidad. Pero más allá del campo médico y de los cuidados paliativos, se debe proporcionar una buena calidad de vida hasta el último momento, para así tener una muerte digna y paz espiritual con él mismo y su entorno. Este curso de introducción a la tanatología resaltó la importancia que tiene esta área en una institución, que en su mayoría atiende a una población de pacientes con enfermedades crónicas, progresivas, degenerativas e incurables y que el equipo de salud este preparado por su perfil profesional a enfrentarse con la muerte, y cómo tratarla cuando es inevitable ya que ésta, es la enemiga número uno y no se les sensibiliza y educa para poder tratar al paciente y a la familia en este proceso inevitable y doloroso.

Palabras clave: tanatología, duelo, duelo complicado.

\section{Impact assessment of an educational intervention in the field of thanatology in INNN}

\begin{abstract}
Objective: to assess whether an educational intervention in the area of thanatology alter the perception of providers of health care in our institution. There is a general ignorance on the subject, so knowing that the Thanatology help the team to provide health care for a patient in a holistic manner, with warmth and quality, going beyond the purely medical and palliative care providing as much as possible a quality of life until the last moment which lead to a dignified death, with spiritual peace with itself and its environment. This introductory course to Thanatology stressed the importance of the area tanatologica an institution like this, most of which serves a population of patients with chronic, progressive, degenerative and incurable, given that the team is ready for his health professional to deal with death, but not when it is treated as inevitable as it is the number one enemy, and they are not sensitized and educated in order to treat the patient and family at this painful and inevitable process.
\end{abstract}

Key words: thanatology, grief, death, dying.

Correspondencia: María del R. Durán Medina. Trabajo Social Instituto Nacional de Neurología y Neurocirugía Manuel Velasco Suárez.

Laboratorio de Investigación Sociomédica. Av. Insurgentes Sur \# 3877. Col. La Fama. 14269. México, D.F.

$\mathrm{S}$ e ha detectado la necesidad de sensibilizar al personal de salud del INNN; con respecto al área de la tanatología, pues los usuarios de este Instituto y familiares experimentan con frecuencia el proceso de duelo anticipatorio y complicado, los cuales requieren de manejo interpersonal específico.

\section{MATERIAL Y MÉTODOS}

Se integró un grupo de prestadores de salud del INNN, (médicos, enfermeras, trabajadores sociales y otros) a los cuales se les aplicó un cuestionario diseñado para evaluar sus percepciones y conceptos en torno a los aspectos tanato- 
lógicos y bioéticos de las enfermedades terminales.

Después se impartió un curso de introducción a la tanatología que duro 3 días (15 horas en promedio). Posterior a la terminación del curso, se aplicó nuevamente el cuestionario, se utilizó estadística descriptiva e ingerencial para conocer las características de la muestra y el grado de modificación de las percepciones en torno al área de tanatología.

\section{Consideraciones metodológicas}

Este cuestionario se aplicó a 45 asistentes al curso de tanatología, el análisis estadístico de estos sólo se pudo realizar de forma exploratoria, al carecer de ambos instrumentos de datos de importancia para realizar el cruce de variables o establecer una asociación entre éstas tales como:

- $\quad$ Edad

- Sexo

- Estado civil

- Escolaridad

- Profesión

- Religión

No obstante, por las limitaciones y naturaleza de las preguntas que integran el cuestionario, todas ellas de tipo abierto, el análisis se trabajo a partir de la realización de categorías, tratando de cerrar las respuestas a palabras que estuvieran asociadas entre sí, por ello sería recomendable en lo futuro diseñar una red semántica o instrumento ad hoc, tomando como antecedente el instrumento que hoy nos ocupa.

\section{RESULTADOS}

\section{Experiencias con la muerte}

El 82.2\% del personal de salud que asistió al curso de tanatología señaló haber estado cerca de un moribundo, lo que les originó sentimientos de tristeza e impotencia. De éstas, el 94.6\% señaló haber proporcionado al moribundo algún tipo de ayuda, de tipo emocional (compañía, consuelo, entre otros) tablas 1,2 y 3 .

El $31.1 \%$ considera que ha estado cerca de morir, de ellos el $64.45 \%$ por haber sufrido un accidente tablas 10 y 11 .

Destaca el $31.2 \%$ al haber pensado en el suicidio a causa de la muerte de un familiar (28.6\%) y sufrir depresión $(21.4 \%)$ tabla 26.

\section{Significados de la muerte}

En cuanto al significado de la muerte, el $28.9 \%$ de los entrevistados asociaron este término con el fin de la vida, en tanto que para el $24.4 \%$ es una fase más de la vida. Para el
$35.6 \%$ las palabras asociadas al significado de su propia muerte, la describieron como término (fin, consumación, conclusión) de la vida, en tanto que para el $11.1 \%$ implica separación y en igual porcentaje desaparecer tablas 6 y 7.

\section{Miedo ante la muerte}

Respecto a la pregunta relacionada con ¿le tiene usted miedo a su muerte?, puede señalarse que Feifel, Eissler, Johnson, Spielberger, Kübler-Ross, entre otros consideran que el pensamiento de la muerte es una fuente de angustia y miedo para el hombre, principalmente porque:

- Se desconoce lo que pasa después de la muerte.

- No podemos, del todo, controlar el cómo, cuándo y dónde morir.

En ese sentido el $44.4 \%$ afirmó tener miedo a la muerte, en lo que respecta a los temores que ésta pueda originar en ese momento, se encuentra que sus temores son por asuntos pendientes (17.8\%), el $13.3 \%$ siente miedo porque su muerte sea dolorosa, en igual porcentaje porque sea inesperada o temor a lo desconocido respectivamente tablas 8 y 9 .

\section{Cómo y cuándo morir}

En lo que respecta a cómo morir, el 35.6\% de los asistentes al curso preferiría que fuera sin sufrimiento, el $24.4 \%$ les gustaría morir cuando estén dormidos; es decir, para la mayoría de los entrevistados la muerte tiene que llegar sin causar ningún dolor tabla 12.

La edad en la que preferirían morir, el $51.1 \%$ señaló que a cualquier edad, el $37.7 \%$ en edades mayores a los 70 años, respuesta lógica si la relacionamos con el significado que dan muchos de ellos a la muerte al considerarla como una etapa final del ciclo de vida tablas 7 y 13 .

En cuanto al lugar en el que preferirían morir, el $86.7 \%$ refirió que en su casa y de ellos el $82.25 \%$ rodeados de su familia tablas 14 y 15 .

Respecto a la forma en que desearían ser sepultados, el $60 \%$ señaló que preferiría ser cremado tabla 16.

El $93.3 \%$ de los entrevistados afirmaron que de llegar a presentar alguna enfermedad mortal considerarían oportuno saber esta situación, ya que el tiempo que les queda de vida lo emplearían disfrutando de la vida, familiares y amigos tabla s 21,22 y 23.

Hoy en diá el avance del conocimiento entorno al funcionamiento del cuerpo humano y al desarrollo cada vez más preciso de tecnología puesto a disposición de la salud de los seres humanos, ha hecho que las maniobras médicas 
permitan prolongar la vida y mejorar la calidad de ésta, al respecto, el 20\% de los encuestados afirmó que en el momento de su muerte no le gustaría que le realizaran ningún tipo de maniobra médica, mientras que al $80 \%$ restante refirió que estaría dispuesta a aprobar que le hicieran las necesarias (66.6\%) tablas 18 y 19.

\section{La incertidumbre ante la muerte}

La principal preocupación que tienen ante la muerte son principalmente, los problemas legales $(42.2 \%)$ que podrían dejar o desarrollar a causa de este hecho, como puede ser propiedades de las cosas (vivienda, coches), divorcios/matrimonios, entre otros, por ello, el 95.5\% señaló que les gustaría realizar su testamento en vida; sin embargo, en ese momento sólo el $11.1 \%$ tenía su testamento hecho, por lo que es necesario la implementación de asesorías legales dirigidas a prevenir este tipo de problemas tablas 17, 28 y 29.

Por otra parte, también plantean que ante la proximidad de la muerte, el $71.1 \%$ preferiría morir antes que alguno de sus seres queridos, en caso de que sucediera lo contrario desean que la muerte de alguno de ellos no fuera dolorosa $(28.9 \%)$ tablas 24 y 25 .

\section{Cuestionario: taller de tanatología}

Tabla 1. ¿Has tenido la oportunidad de estar cerca de un moribundo?

\begin{tabular}{c|c|c}
\hline & Frecuencia & \% \\
\hline $\mathrm{Si}$ & 37 & 82.2 \\
No & 8 & 17.8 \\
Total & 45 & 100.0 \\
\hline \multicolumn{2}{|c}{ Fuente: cuestionario aplicado en el curso de tanatología a }
\end{tabular}
personal del INNN. Julio del 2007.

Tabla 2. ¿Qué tipo de emociones experimentó?.

\begin{tabular}{|c|c|c|}
\hline & Frecuencia & $\%$ \\
\hline Amor & 3 & 6.7 \\
\hline Ansiedad & 2 & 4.4 \\
\hline Angustia & 3 & 6.7 \\
\hline Compasión & 1 & 2.2 \\
\hline Coraje & 3 & 6.7 \\
\hline Depresión & 1 & 2.2 \\
\hline Desesperación & 1 & 2.2 \\
\hline Dolor & 2 & 4.4 \\
\hline Frustración & 3 & 6.7 \\
\hline Impotencia & 5 & 11.1 \\
\hline Miedo & 1 & 2.2 \\
\hline Pesar & 1 & 2,2 \\
\hline Tristeza & 8 & 17.8 \\
\hline Tensión & 1 & 2.2 \\
\hline No Aplica & 9 & 20.0 \\
\hline No Contestó & 1 & 2.2 \\
\hline Total & 45 & 100.0 \\
\hline
\end{tabular}

Tabla 3. ¿Le proporcionó ayuda, asistencia, compañía?.

\begin{tabular}{c|c|c}
\hline \multicolumn{2}{c|}{ Frecuencia } & $\%$ \\
\hline $\mathrm{Si}$ & 35 & 94.6 \\
No & 2 & 5.4 \\
Total & 37 & 100.0 \\
\hline
\end{tabular}

Fuente: cuestionario aplicado en el curso de tanatología a personal del INNN. Julio del 2007.

Tabla 4. ¿Qué tipo de ayuda?.

\begin{tabular}{l|c|c}
\hline & Frecuencia & $\%$ \\
\hline $\begin{array}{l}\text { Médica } \\
\text { (primeros auxilios, confort) }\end{array}$ & 10 & 27.0 \\
\cline { 1 - 1 } $\begin{array}{l}\text { Emocional } \\
\text { (amistad, consuelo, }\end{array}$ & 23 & 62.2 \\
compañía, asistencia) & 4 & 10.8 \\
$\begin{array}{l}\text { No contestó } \\
\text { Total }\end{array}$ & 37 & 100.0 \\
\hline $\begin{array}{l}\text { Fuente: cuestionario aplicado en el curso de tanatología a personal del INNN. Julio del } \\
\text { 2007. }\end{array}$
\end{tabular}

Tabla 5. ¿Considera usted que esta preparado para ofrecer apoyo a una persona que va a morir?.

\begin{tabular}{l|c|c}
\hline & Frecuencia & $\%$ \\
\hline $\mathrm{Si}$ & 12 & 26.7 \\
No & 33 & 73.3 \\
Total & 45 & 100.0 \\
\hline \multicolumn{2}{|c}{ Fuente: cuestionario aplicado en el curso de tanatología a }
\end{tabular}
personal del INNN. Julio del 2007.

Tabla 6. ¿En general, cuál es el significado de la muerte?.

\begin{tabular}{l|c|c}
\hline & Frecuencia & $\%$ \\
\hline $\begin{array}{l}\text { Fase de la vida } \\
\text { (parte de la vida) }\end{array}$ & 11 & 24.4 \\
$\begin{array}{l}\text { Fin de la vida } \\
\text { (se termina) }\end{array}$ & 13 & 28.9 \\
Perdida del cuerpo & 3 & 6.7 \\
físico(dejar de existir) & & \\
Orgánica/cerebral & 6 & 13.3 \\
Perdida & 1 & 2.2 \\
Dolorosa & 2 & 4.4 \\
Descanso & 3 & 6.7 \\
Reencarnación & 6 & 13.3 \\
Total & 45 & 100.0 \\
\hline Fuente: cuestionario aplicado en el curso de tanatología a personal del
\end{tabular}

\section{DISCUSIÓN}

$\mathrm{Al}$ inicio del curso el $31.1 \%$ de los asistentes, afirmó que asistía con la finalidad de poder tener una mejor compresión del proceso de la muerte, lo que les permitiría disfrutar más de la vida, al considerar que la tanatología puede contribuir, principalmente al entendimiento de está y del proceso de duelo, no sólo para su vida personal, sino también para el servicio que brindan en el INNN. 
Tabla 7. ¿Cuál es el significado de su muerte?.

\begin{tabular}{|c|c|c|}
\hline & Frecuencia & $\%$ \\
\hline $\begin{array}{l}\text { Término } \\
\text { (fin, conclusión, } \\
\text { consumación) }\end{array}$ & 16 & 35.6 \\
\hline $\begin{array}{l}\text { Inevitable/ sin } \\
\text { retorno /no } \\
\text { aceptación }\end{array}$ & 2 & 4.4 \\
\hline Dolor & 2 & 4.4 \\
\hline Miedo & 2 & 4.4 \\
\hline Lo peor & 1 & 2.2 \\
\hline Desaparecer & 5 & 11.1 \\
\hline Trascendencia & 2 & 4.4 \\
\hline Tranquilidad & 2 & 4.4 \\
\hline Separación & 5 & 11.1 \\
\hline No contestó & 8 & 17.8 \\
\hline Total & 45 & 100.0 \\
\hline
\end{tabular}

Tabla 8. ¿Le tiene miedo a su muerte?.

\begin{tabular}{l|c|c}
\hline & Frecuencia & $\%$ \\
\hline & 20 & 44.4 \\
No & 25 & 55.6 \\
Total & 45 & 100.0 \\
\hline
\end{tabular}

Fuente: cuestionario aplicado en el curso de tanatología a personal del INNN. Julio del 2007.

Tabla 9. ¿A qué teiene usted miedo en el momento de su muerte?

\begin{tabular}{l|c|c}
\hline & Frecuencia & $\%$ \\
\hline A qué sea dolorosa & 6 & 13.3 \\
Inesperado & 6 & 13.3 \\
No ver a mis seres queridos & 3 & 6,7 \\
Pendientes & 8 & 17.8 \\
Desconocido & 6 & 13.3 \\
Al no haber vivido & 4 & 8.9 \\
Dejar en desamparo & 2 & 4.4 \\
(familia, hijos, etc.) & 5 & 11.1 \\
A nada & 5 & 11.1 \\
No contestó & 45 & 100.0 \\
\hline Total & & \\
\hline \multicolumn{2}{l|}{ Fuente: cuestionario aplicado en el curso de tanatología a personal del INNN. Julio del } \\
2007.
\end{tabular}

Tabla 10. ¿Considera usted que alguna vez ha estado cerca de la muerte?.

\begin{tabular}{l|c|c}
\hline & Frecuencia & $\%$ \\
\hline $\mathrm{Si}$ & 14 & 31.1 \\
No & 31 & 68.9 \\
Total & 45 & 100.0 \\
\hline
\end{tabular}

Fuente: cuestionario aplicado en el curso de tanatología a personal del INNN. Julio del 2007.
Tabla 11. Describa su experiencia.

\begin{tabular}{l|c|c}
\hline & Frecuencia & $\%$ \\
\hline Durante el nacimiento & 1 & 7.1 \\
Accidente & 9 & 64.4 \\
Amenaza con arma de & 1 & 7.1 \\
fuego & & \\
En avión & 1 & 14.2 \\
Por su profesión & 14 & 7.1 \\
\multicolumn{2}{|c}{ Total } & 100 \\
\hline \multicolumn{2}{l|}{ Fuente: cuestionario aplicado en el curso de tanatologia a personal del INNN. Julio del }
\end{tabular}

2007

Tabla 12. ¿Cómo preferiría morir?.

\begin{tabular}{l|c|c}
\hline & Frecuencia & $\%$ \\
\hline Dormida & 11 & 24.4 \\
Sin sufrimiento & 16 & 35.6 \\
Solo & 1 & 2.2 \\
Feliz & 5 & 11.1 \\
Infarto & 5 & 11.1 \\
Consciente & 2 & 4.4 \\
No sabe & 5 & 11.1 \\
Total & 45 & 100.0 \\
\hline Fuente: cuestionario aplicado en el curso de tanatología a personal
\end{tabular}
del INNN. Julio del 2007.

Tabla 13. ¿A que edad?

\begin{tabular}{l|c|c}
\hline & Frecuencia & $\%$ \\
\hline 50 años & 1 & 2.2 \\
60 años & 2 & 4.4 \\
65 años & 2 & 4.4 \\
70 años & 7 & 15.6 \\
75 años & 2 & 4.4 \\
80 años & 6 & 13.3 \\
85 años & 1 & 2.2 \\
100 años & 1 & 2.2 \\
Cualquier edad & 23 & 51.1 \\
Total & 45 & 100.0 \\
\hline Fuente: cuestionario aplicado en el curso de tanatologia a personal del \\
INNN. Julio del 2007.
\end{tabular}

Al finalizar el curso, el $82.9 \%$ de los asistentes refirió que su percepción de la muerte se modificó a través del curso, al considerar la muerte como «algo natural» $(31.0 \%)$. Cfr. Tabla 35,36 .

En cuanto a la percepción del curso, $85.7 \%$ opino que fue bueno e interesante, cabe señalar que el Instituto es el primero impartir este tipo, aunque un $5.7 \%$ consideró que fue poco dinámico y didáctico, esta última opinión se enfatiza con el $25.7 \%$ que sugiere que el próximo sea más didáctico, a través de su desarrollo en la modalidad de taller, por lo que se tendría que recurrir a técnicas y material impreso tablas 47 y 48 . 
Tabla 14. ¿En donde prefiria morir?.

\begin{tabular}{l|c|c}
\hline & Frecuencia & \\
\hline Trabajo & 1 & 2.2 \\
Casa & 39 & 86.7 \\
Hospital & 1 & 2.2 \\
En cama & 2 & 4.4 \\
Donde sea & 2 & 4.4 \\
Total & 45 & 100.0 \\
\hline
\end{tabular}

Fuente: cuestionario aplicado en el curso de tanatología a personal del INNN. Julio del 2007

Tabla 15. ¿Quién le gustaría que le acompañará antes de morir?.

\begin{tabular}{l|c|c}
\hline & Frecuencia & $\%$ \\
\hline Familia & 29 & 64.4 \\
Amigos & 3 & 6.7 \\
Hijos & 1 & 2.2 \\
Pareja & 7 & 15.6 \\
Nadie & 3 & 6.7 \\
No contestó & 2 & 4.4 \\
Total & 45 & 100.0 \\
\hline Fuente: cuestionario aplicado en el curso de tanatología a
\end{tabular}
personal del INNN. Julio del 2007

Tabla 16. Al morir ¿qué desea que se haga con su cuerpo?.

\begin{tabular}{l|c|c}
\hline & Frecuencia & $\%$ \\
\hline Sepultado & 15 & 33.3 \\
Cremado & 27 & 60.0 \\
No sabe & 3 & 6.7 \\
Total & 45 & 100.0 \\
\hline Fuente: cuestionario aplicado en el curso de tanatologia a
\end{tabular}

Fuente: cuestionario aplicado en el curso de tanatología

Tabla 17. ¿Qué le gustaría hacer para resolver cosas pendientes antes de morir?.

\begin{tabular}{l|c|c}
\hline & Frecuencia & $\%$ \\
\hline Testamento & 5 & 11.1 \\
Despedirme & 2 & 4.4 \\
Platicar con familiares & 6 & 13.3 \\
Visitar amigos & 1 & 2.2 \\
Resolver problemas legales & 19 & 42,2 \\
Apurarme & 2 & 4.4 \\
Vivir la vida & 3 & 6.7 \\
No contestó & 7 & 15.6 \\
Total & 45 & 100.0 \\
\hline \multicolumn{2}{|c|}{ Fuente: cuestionario aplicado en el curso de tanatología a personal del INNN. }
\end{tabular}

en el curso de tanatología a personal del INNN. Julio del 2007.

Tabla 18. En el momento de estar muriendo, ¿le gustaría que le realizaran algunas maniobras médicas?.

\begin{tabular}{l|c|c}
\hline & Frecuencia & $\%$ \\
\hline $\mathrm{Si}$ & 9 & 20.0 \\
$\mathrm{No}$ & 36 & 80.0 \\
Total & 45 & 100.0 \\
\hline Fuente: cuestionario aplicado en el curso de \\
tanatologia a personal del INNN. Julio del 2007.
\end{tabular}

tanatologianorsonaldel INNN Julio del 2007.
Tabla 19. ¿Cuáles?.

\begin{tabular}{l|c|c}
\hline & Frecuencia & $\%$ \\
\hline Extracción y donación de órganos & 1 & 11.1 \\
Las necesarias & 6 & 66.6 \\
Depende las circunstancias & 2 & 22.2 \\
Total & 9 & 100.0 \\
\hline
\end{tabular}

Fuente: cuestionario aplicado en el curso de tanatología a personal del INNN. Julio del 2007.

Tabla 20. En el moemento de su fase terminal, ¿qué le gustaría que hicieran sus familiares?

\begin{tabular}{l|c|c}
\hline & Frecuencia & $\%$ \\
\hline Compañía & 20 & 44.4 \\
Conversar & 2 & 4.4 \\
Rezar & 3 & 6.7 \\
Donar órganos & 1 & 2.2 \\
Dejarme ir & 12 & 26.7 \\
Nada & 6 & 13.3 \\
No contesto & 1 & 2.2 \\
Total & 45 & 100.0 \\
\hline \multicolumn{2}{l|}{ Fuente: cuestionario aplicado en el curso de tanatología a personal del }
\end{tabular}
INNN. Julio del 2007.

Tabla 21. Si tuviera una enfermedad mortal, ¿le gustaría saber la verdad?.

\begin{tabular}{l|c|c}
\hline & Frecuencia & $\%$ \\
\hline $\mathrm{Si}$ & 42 & 93.3 \\
No & 3 & 6.7 \\
Total & 45 & 100.0 \\
\hline \multicolumn{2}{l}{$\begin{array}{l}\text { Fuente: cuestionario aplicado en el curso de } \\
\text { tanatología a personal del INNN. Julio del } 2007 .\end{array}$}
\end{tabular}

Tabla 22. ¿Le gustaría saber su enfermedad cuanto tiempo le queda de vida?.

\begin{tabular}{l|c|c}
\hline & Frecuencia & $\%$ \\
\hline Si & 36 & 80.0 \\
No & 9 & 20.0 \\
Total & 45 & 100.0 \\
\hline \multicolumn{2}{|c|}{ Fuente: cuestionario aplicado en el curso de }
\end{tabular}

tanatología a personal del INNN. Julio del 2007.

Tabla 23. ¿Cómo lo emplearía?.

\begin{tabular}{l|c|c}
\hline & Frecuencia & $\%$ \\
\hline Serenidad & 1 & 2.2 \\
Haciendo lo pendiente & 4 & 8.9 \\
Disfrutando & 21 & 46.7 \\
(familia, vida, amistades) & & \\
Cerrando ciclos & 3 & 6.7 \\
Hacer lo mejor & 5 & 11.1 \\
No contestó & 11 & 24.4 \\
Total & 45 & 100.0 \\
\hline
\end{tabular}

Fuente: cuestionario aplicado en el curso de tanatología a personal del INNN. Julio del 2007. 
Enf Neurol (Mex)

Tabla 24. Ante la proximidad de la muerte ¿quién prefiere que muera primero: usted o su ser más querido?

\begin{tabular}{l|c|c}
\hline & Frecuencia & $\%$ \\
\hline Yo & 32 & 71.1 \\
Al destino & 10 & 22.2 \\
No lo se & 3 & 6.7 \\
Total & 45 & 100.0 \\
\hline Fuente: cuestionario aplicado en el curso de tanatología a \\
personal del INNN. Julio del 2007.
\end{tabular}
sería la forma menos dolorosa para ambos?

\begin{tabular}{l|c|c}
\hline & Frecuencia & $\%$ \\
\hline Resignación & 9 & 20.0 \\
No sufriera & 13 & 28.9 \\
Recordar & 3 & 6.7 \\
Superarlo & 1 & 2.2 \\
Compañia & 5 & 11.1 \\
Planificado & 6 & 13.3 \\
No lo sé & 8 & 17.8 \\
Total & 45 & 100.0 \\
\hline Fuente: cuestionario aplicado en el curso de tanatología a personal
\end{tabular}

Tabla 26. ¿Ha pensado alguna vez en el suicidio?

\begin{tabular}{l|c|c}
\hline & Frecuencia & $\%$ \\
\hline $\mathrm{Si}$ & 14 & 31.2 \\
$\mathrm{No}$ & 31 & 68.8 \\
Total & 45 & 100.0 \\
\hline \multicolumn{2}{|c}{ Fuente: cuestionario aplicado en el curso de tanatología a } \\
personal del INNN. Julio del 2007.
\end{tabular}

Tabla 27. ¿Cuál ha sido la causa que lo ha hecho pensar en quitarse la vida?.

\begin{tabular}{l|c|c}
\hline & Frecuencia & $\%$ \\
\hline Culpabilidad & 1 & 7.1 \\
Problemas familiares & 2 & 14.3 \\
Depresión & 3 & 21.4 \\
Divorcio & 2 & 14.3 \\
Muerte de un familiar & 4 & 28.6 \\
Reflexionar & 2 & 14.3 \\
Total & 14 & 100.0 \\
\hline
\end{tabular}

Fuente: cuestionario aplicado en el curso de tanatología a personal del INNN. Julio del 2007.

Tabla 28. ¿Tiene usted hecho su testamento?.

\begin{tabular}{l|c|c}
\hline & Frecuencia & \\
\hline $\mathrm{Si}$ & 5 & 11.1 \\
No & 40 & 88.9 \\
Total & 45 & 100.0 \\
\hline
\end{tabular}

Fuente: cuestionario aplicado en el curso de tanatología a personal del INNN. Julio del 2007
Tabla 29. En algún momento le gustaría hacer un testamento señalando lo que quiere que se haga con usted en los últimos momentos antes de morir.

\begin{tabular}{l|c|c}
\hline & Frecuencia & $\%$ \\
\hline $\mathrm{Si}$ & 43 & 95.6 \\
No & 2 & 4.4 \\
Total & 45 & 100.0 \\
\hline \multicolumn{2}{|c}{ Fuente: cuestionario aplicado en el curso de } \\
tanatologia a personal del INNN. Julio del 2007.
\end{tabular}

Tabla 30. ¿Cuál es su emoción domínante mientras llena el custionario

\begin{tabular}{l|c|c}
\hline & Frecuencia & $\%$ \\
\hline Tranquilidad & 4 & 8.9 \\
Incertidumbre & 10 & 22.2 \\
Duda & 1 & 2.2 \\
Deseo de vivir cada minuto & 1 & 2.2 \\
No estoy preparado para & 5 & 11.1 \\
la muerte & & \\
Confusa & 4 & 8.9 \\
Reflexión & 3 & 6.7 \\
Tristeza & 3 & 6.7 \\
$\quad$ Melancolía & 3 & 6.7 \\
Seguridad & 2 & 4.4 \\
Ansiedad & 2 & 4.4 \\
Temor & 1 & 2.2 \\
No contestó & 6 & 13.3 \\
Total & 45 & 100.0 \\
\hline
\end{tabular}

Fuente: cuestionario aplicado en el curso de tanatología a personal del INNN. Julio del 2007.

Tabla 31. ¿Le gustaría tener epitafio?

\begin{tabular}{l|c|c}
\hline & Frecuencia & $\%$ \\
\hline $\mathrm{Si}$ & 22 & 48.9 \\
No & 16 & 35.6 \\
No contestó & 7 & 15.6 \\
Total & 45 & 100.0 \\
\hline
\end{tabular}
Fuente: cuestionario aplicado en el curso de tanatología a

Tabla 32. ¿Está usted en este taller por miedo de la muerte?

\begin{tabular}{l|c|c}
\hline & Frecuencia & $\%$ \\
\hline $\mathrm{Si}$ & 5 & 11.1 \\
$\mathrm{No}$ & 40 & 88.9 \\
Total & 45 & 100.0 \\
\hline \multicolumn{2}{|c}{$\begin{array}{l}\text { Fuente: cuestionario aplicado en el curso de } \\
\text { tanatologia a personal del INNN. Julio del 2007. }\end{array}$}
\end{tabular}

Tabla 33. ¿Cree usted que el estudio de la tanatología puede ayudarle algo?

\begin{tabular}{l|c|c}
\hline & Frecuencia & $\%$ \\
\hline $\mathrm{Si}$ & 43 & 95.6 \\
No & 2 & 4.4 \\
Total & 45 & 100.0 \\
\hline $\begin{array}{l}\text { Fuente: } \text { cuestionario aplicado en el curso de } \\
\text { tanatologia a personal del INNN. Julio del 2007. }\end{array}$
\end{tabular}


Tabla 34. ¿Por qué?.

\begin{tabular}{l|c|c}
\hline & Frecuencia & $\%$ \\
\hline Ayudar al buen morir & 11 & 24.4 \\
Comprensión & 14 & 31.1 \\
Manejar el proceso & 11 & 24.4 \\
El duelo & 6 & 13.3 \\
No contestó & 3 & 6.7 \\
Total & 45 & 100.0 \\
\hline
\end{tabular}

Fuente: cuestionario aplicado en el curso de tanatología a personal del INNN. Julio del 2007.

Tabla 35. ¿Se ha modificado su percepción y concepto de muerte con este curso?.

\begin{tabular}{l|c|c}
\hline & Frecuencia & \\
\hline $\mathrm{Si}$ & 29 & 82.9 \\
$\mathrm{No}$ & 6 & 17.1 \\
Total & 35 & 100 \\
\hline $\begin{array}{l}\text { Fuente: cuestionario aplicado en el curso de tanatología a } \\
\text { personal del INNN. Julio del 2007. }\end{array}$
\end{tabular}

Tabla 36. ¿Por qué si?.

\begin{tabular}{|c|c|c|}
\hline & Frecuencia & $\%$ \\
\hline $\begin{array}{l}\text { Importancia de morir con } \\
\text { dignidad }\end{array}$ & 1 & 3.4 \\
\hline $\begin{array}{l}\text { Se percibe la muerte como } \\
\text { algo natural }\end{array}$ & 9 & 31.0 \\
\hline Aceptar el proceso & 5 & 17.2 \\
\hline $\begin{array}{l}\text { Importancia del apoyo } \\
\text { familiar }\end{array}$ & 2 & 6.8 \\
\hline Hubo gran reflexión & 6 & 20.6 \\
\hline Ya era de mi conocimiento & 6 & 20.6 \\
\hline Total & 29 & 100 \\
\hline
\end{tabular}

2007.

Tabla 37. ¿Cree que es importante el trabajo de tanatología en nuestra institución?.

\begin{tabular}{l|c|c}
\hline & Frecuencia & \\
\hline $\mathrm{Si}$ & 35 & 100 \\
\hline $\begin{array}{l}\text { Fuente: cuestionario aplicado en el curso de tanatología a } \\
\text { personal del INNN. Julio del 2007. }\end{array}$
\end{tabular}

Tabla 38. ¿Por qué si?.

\begin{tabular}{l|c|c}
\hline & Frecuencia & $\%$ \\
\hline Manejo del duelo & 10 & 28.6 \\
$\begin{array}{l}\text { Mejorar la calidad } \\
\text { del servicio }\end{array}$ & 14 & 40.0 \\
$\begin{array}{l}\text { Orientación al paciente } \\
\text { y su familia }\end{array}$ & 6 & 17.1 \\
No contestó & 5 & 14.3 \\
Total & 35 & 100 \\
\hline $\begin{array}{l}\text { Fuente: cuestionario aplicado en el curso de tanatología a personal del INNN. Julio } \\
\text { del 2007. }\end{array}$
\end{tabular}

Tabla 39. ¿Recurriría al trabajo tanatológico?

\begin{tabular}{l|c|c}
\hline & Frecuencia & $\%$ \\
\hline $\mathrm{Si}$ & 34 & 97.1 \\
$\mathrm{No}$ & 1 & 2.9 \\
Total & 35 & 100 \\
\hline \multicolumn{2}{|c}{ Fuente: cuestionario aplicado en el curso de tanatología a } \\
personal del INNN. Julio del 2007.
\end{tabular}

Tabla 40. ¿Por qué si?.

\begin{tabular}{l|c|c}
\hline & Frecuencia & $\%$ \\
\hline Manejo de duelo & 10 & 29.4 \\
$\begin{array}{l}\text { Oportunidad de cerrar } \\
\text { ciclos afectivos }\end{array}$ & 11 & 32.3 \\
Resolver pendientes & 6 & 17.6 \\
Mejorar la calidad del & 7 & 20.5 \\
servicio & 34 & 100 \\
Total & & \\
\hline
\end{tabular}

Fuente: cuestionario aplicado en el curso de tanatología a personal del INNN. Julio del 2007.

Tabla 41. Características sociodemográficas de los asistentes al grupo de apoyo tanatológico

\begin{tabular}{|c|c|}
\hline Variable & $\begin{array}{c}\text { Porcentaje } \\
\text { n (69) }\end{array}$ \\
\hline \multicolumn{2}{|l|}{ Edad } \\
\hline $15-30$ & 14.5 \\
\hline $31-40$ & 21.7 \\
\hline $41-50$ & 36.2 \\
\hline $51-60$ & 17.4 \\
\hline+60 & 10.1 \\
\hline \multicolumn{2}{|l|}{ Sexo } \\
\hline Femenino & 72.9 \\
\hline Masculino & 27.1 \\
\hline \multicolumn{2}{|l|}{ Escolaridad } \\
\hline Ninguna & 4.3 \\
\hline Básica (1-9) & 53.6 \\
\hline Bachillerato (10-12) & 21.7 \\
\hline Profesionista (13-17) & 20.3 \\
\hline \multicolumn{2}{|l|}{ Tiempo con enf. } \\
\hline-1 mes & 49.2 \\
\hline $1-12$ meses & 20.3 \\
\hline+12 meses & 30.5 \\
\hline \multicolumn{2}{|l|}{ Servicio } \\
\hline UTI & 45.2 \\
\hline Urgencias & 12.9 \\
\hline $\mathrm{UCl}$ & 9.7 \\
\hline Neurología & 11.3 \\
\hline Neurocirugía & 14.5 \\
\hline Neuropsiq. & 1.6 \\
\hline C. Ext. & 4.8 \\
\hline
\end{tabular}


Tabla 42. ¿Lo recomendaría a amigos o conocidos, si ellos fueran afectados por una enfermedad terminal o duelo?.

\begin{tabular}{l|c|c}
\hline & Frecuencia & $\%$ \\
\hline $\mathrm{Si}$ & 27 & 77.1 \\
$\mathrm{No}$ & 8 & 22.8 \\
Total & 35 & 100 \\
\multicolumn{2}{|c}{ Fuente: cuestionario aplicado en el curso de tanatología } \\
a personal del INNN. Julio del 2007.
\end{tabular}

Tabla 43. ¿Por qué si?

\begin{tabular}{l|c|c}
\hline & Frecuencia & $\%$ \\
\hline Manejo de duelo & 17 & 62.9 \\
$\begin{array}{l}\text { Oportunidad de cierre de } \\
\text { ciclos afectivos }\end{array}$ & 5 & 18.5 \\
$\begin{array}{l}\text { Oportunidad de mejorar la } \\
\text { calidad de vida }\end{array}$ & 5 & 18.5 \\
\hline Total & 27 & 100 \\
\hline
\end{tabular}

Tabla 44. ¿Le gustaría ampliar sus conocimientos sobre tanatología?.

\begin{tabular}{l|c|c}
\hline & Frecuencia & $\%$ \\
\hline $\mathrm{Si}$ & 30 & 85.7 \\
$\mathrm{No}$ & 5 & 14.2 \\
Total & 35 & 100 \\
\hline \multicolumn{2}{|c}{$\begin{array}{l}\text { Fuente: cuestionario aplicado en el curso de tanatología a } \\
\text { personal del INNN. Julio del 2007. }\end{array}$}
\end{tabular}

Tabla 45. ¿Por qué si?.

\begin{tabular}{l|c|c}
\hline & Frecuencia & $\%$ \\
\hline $\begin{array}{l}\text { Mejorar la calidad del } \\
\text { servicio }\end{array}$ & 4 & 13.3 \\
$\begin{array}{l}\text { Brindar apoyo al que lo } \\
\text { necesite }\end{array}$ & 8 & 26.6 \\
Manejo de duelo & 5 & 16.6 \\
Tema de interés & 13 & 43.3 \\
Total & 30 & 100 \\
\hline
\end{tabular}

Tabla 46. Medias y desviaciones de los asistentes al grupo de apoyo tanatológico, por pruebas aplicadas.

\begin{tabular}{|c|c|c|c|}
\hline Variable & Depresión & Ansiedad & Estrés \\
\hline \multicolumn{4}{|l|}{ Sexo } \\
\hline Femenino & $9.13 .7(0-16)$ & $7.54 .5(0-17)$ & $31.49 .9(0-47)$ \\
\hline Masculino & $8.84 .2(0-16)$ & $7.54 .5(0-15)$ & $27.813 .0(0-41)$ \\
\hline \multicolumn{4}{|l|}{ Edad } \\
\hline $15-40$ & $9.63 .0(4-15)$ & $8.03 .7(2-17)$ & $31.57 .8(0-41)$ \\
\hline $41-50$ & $8.54 .2(0-16)$ & $7.04 .2(0-15)$ & $31.98 .6(0-43)$ \\
\hline+50 & $8.74 .2(0-16)$ & $7.55 .8(0-17)$ & $28.714 .5(0-47)$ \\
\hline \multicolumn{4}{|l|}{ Escolaridad } \\
\hline $0-9$ & $8.14 .7(0-16)$ & $8.35 .0(0-17)$ & $30.510 .7(0-47)$ \\
\hline $10-17$ & $10.33 .0(4-16)$ & $6.43 .4(2-15)$ & $31.49 .7(0-41)$ \\
\hline
\end{tabular}

Tabla 47. Opinión sobre el curso.

\begin{tabular}{l|c|c}
\hline & Frecuencia & $\%$ \\
\hline Curso excelente & 14 & 40.0 \\
Muy bien, muy interesante & 16 & 45.7 \\
Debe continuar & 1 & 2.9 \\
Poco dinámico y didáctico & 2 & 5.7 \\
Enriquecedor & 2 & 5.7 \\
Total & 35 & 100 \\
\hline Fuente: cuestionario aplicado en el curso de tanatología a personal del INNN. Julio del \\
2007.
\end{tabular}

Tabla 48. ¿Qué nos podría sugerir para enriquecer este curso?

\begin{tabular}{l|c|c}
\hline & Frecuencia & $\%$ \\
\hline Mayor duración (curso y ponencias) & 4 & 11.4 \\
Mayor difusión & 3 & 8.6 \\
Nuevos temas y cursos a fines & 9 & 25.7 \\
Prohibir celulares & 1 & 2.9 \\
Invitar a más • rofesionales & 2 & 5.7 \\
Desarrollo de técnicas, talleres & 9 & 25.7 \\
y uso de material didáctico & & \\
Lugar más amplio & 1 & 2.9 \\
No contestó & 6 & 17.1 \\
Total & 35 & 100 \\
\hline
\end{tabular}

\section{CONCLUSIONES}

Es importante establecer acciones educativas en el área de la tanatología en el INNN; para sensibilizar a los prestadores de servicios de salud e incrementar su capacidad de manejo interpersonal ante problemas de duelo anticipado y duelo complicado ya que esto podría traducirse en una mejora de la calidad de atención en la Institución.

Impartir 4 talleres al año dirigidos a los prestadores de servicio de salud del INNN, con la finalidad de sensibilizar, concienciar sobre la importancia de la tanatología en la institución.

Invitar a ponentes de otras instituciones expertas en el tema para los diferentes talleres y trabajar con los propios duelos para ayudar a bajar la resistencia, miedo y angustia que pueda generarle al personal del equipo de salud el enfrentarse día a día con la muerte de los pacientes y como dar contención y apoyo al paciente y familiares.

\section{BIBLIOGRAFÍA CONSULTADA}

1. Sahún Fray Bernardino. Historia de las cosas de Nueva España Ed. Porrúa, México, 1956.

2. Reyes Zubiría L Alfonso. Acercamientos tanatológico al enfermo terminal y a su familia. Ed. Triple A. Diseño, S.A. de C.V. México 1996.

3. Cavaría R, García G. y Prusquia M. Tanatología, aspectos sociales, 
éticos y legislación. Ed. Talleres Gráficos de la Cámara de Diputados, México 2004.

4. Roas C, Marinelli S, Velez C. Montes de Oca B. Cuidado del enfermo crónico y terminal. Ed. Centro San Camilo, México 2004.

5. Kübler-Ross E. La rueda de la vida. Ed. Zeta, 1997.
6. Kübler-Ross E. Los niños y la muerte. Ed. Luciérnaga S.L. Barcelona España segunda reimpresión 1996.

7. Ariés Ph. El hombre ante la muerte Ed. Taurus, Madrid,1992.

8. Louis Vincent Thomas. Antropología de la muerte Ed. Fondo de Cultura Económica, México 1983. 